\title{
Effect of Rotation on Thermal Instability in Rivlin-Ericksen Elastico-Viscous Fluid
}

\author{
R. C. Sharma and P. Kumar \\ Department of Mathematics, Himachal Pradesh University, Summer Hill, Shimla-171 005, India
}

Z. Naturforsch. 51 a, $821-824$ (1996); received December 27, 1995

\begin{abstract}
The thermal instability of a layer of Rivlin-Ericksen elastico-viscous fluid acted on by a uniform rotation is considered. For stationary convection, a Rivlin-Ericksen elastico-viscous fluid behaves like a Newtonian fluid. It is found that rotation has a stabilizing effect and introduces oscillatory modes in the system. The visco-elasticity also introduces oscillatory modes in the system. A sufficient condition for the non-existence of overstability is also obtained.
\end{abstract}

\section{Introduction}

The effect of thermal instability of a fluid layer acted on by a uniform rotation has been discussed exhaustively by Chandrasekhar [1]. Bhatia and Steiner [2] have studied the problem of thermal instability of a viscoelastic (Maxwell) fluid in the presence of rotation and have found that the rotation has a destabilizing influence in contrast to the stabilizing effect on an ordinary fluid (Newtonian fluid). Sharma [3] has studied the thermal instability of a layer of viscoelastic (Oldroydian) fluid acted on by a uniform rotation and found that rotation has destabilizing as well as stabilizing effects under certain conditions, in contrast to that of a Maxwell fluid where it has a destabilizing effect.

There are many elastico-viscous fluids that cannot be characterized by Maxwell's constitutive relations or Oldroyd's [4] constitutive relations. One such class of elastico-viscous fluids is Rivlin-Ericksen fluid. Srivastava and Singh [5] have studied the unsteady flow of a dusty elastico-viscous Rivlin-Ericksen fluid through channels of different cross-sections in the presence of a time dependent pressure gradient. In another study Garg et al. [6] have studied the rectilinear oscillations of a sphere along its diameter in a conducting dusty Rivlin-Ericksen fluid in the presence of a uniform magnetic field. It is this class of elasticoviscous fluids we are interested in, particularly in the thermal convection in such fluids in the presence of rotation.

Reprint requests to Herrn Dr. R. C. Sharma.
The present paper, therefore, attempts to study the thermal instability in a Rivlin-Ericksen fluid in presence of uniform rotation.

\section{Formulation of the Problem and Dispersion Relation}

Here we consider an infinite horizontal layer of a Rivlin-Ericksen elastico-viscous fluid of depth $d$, which is acted on by a uniform rotation $\boldsymbol{\Omega}(0,0, \Omega)$ and gravity force $g(0,0,-g)$. This layer is heated from below so that a steady adverse temperature gradient $\beta(=|\mathrm{d} T / \mathrm{d} z|)$ is maintained. The equations of motion, continuity and heat conduction governing the flow are

$$
\begin{aligned}
& \frac{\partial \boldsymbol{q}}{\partial t}+(\boldsymbol{q} \cdot \nabla) \boldsymbol{q}=-\frac{1}{\varrho_{0}} \nabla p \\
& +\left(v+v^{\prime} \frac{\partial}{\partial t}\right) \nabla^{2} \boldsymbol{q}+\boldsymbol{g}\left(1+\frac{\delta \varrho}{\varrho_{0}}\right)+2(\boldsymbol{q} \times \boldsymbol{\Omega}) \\
& \nabla \cdot \boldsymbol{q}=0 \\
& \frac{\partial T}{\partial t}+(\boldsymbol{q} \cdot \nabla) T=\kappa \nabla^{2} T
\end{aligned}
$$

where $\boldsymbol{q}(u, v, w), p, \varrho, T, v, v^{\prime}$ and $\kappa$ are respectively the velocity, pressure, density, temperature, kinematic coefficient of viscosity, kinematic viscoelasticity and thermal diffusivity. The equation of state for the fluid is

$$
\varrho=\varrho_{0}\left[1-\alpha\left(T-T_{0}\right)\right],
$$

where $\varrho_{0}, T_{0}$ are respectively, the density and temperature of the fluid at the reference level $z=0$, and $\alpha$ is the coefficient of thermal expansion. 
Thermal Instability in Rivlin-Ericksen Elastico-Viscous Fluid

Here we make the Boussinesq approximation under which the density changes may be disregarded in all terms in the equations of motion except the one in the external force. The initial state is one in which the velocity, density, pressure and temperature at any point in the fluid are respectively given by

$$
\begin{array}{ll}
\boldsymbol{q}=(0,0,0), & \varrho=\varrho(z), \\
p=p(z) \quad \text { and } & T=T(z) .
\end{array}
$$

Let $\boldsymbol{q}(u, v, w), \delta \varrho, \delta p$ and $\theta$ denote, respectively, the perturbations in velocity $(0,0,0)$, density $\varrho$, pressure $p$ and temperature $T$. Then the linearized perturbation equations are

$$
\begin{aligned}
& \frac{\partial \boldsymbol{q}}{\partial t}=-\frac{1}{\varrho_{0}} \nabla \delta p+\left(v+v^{\prime} \frac{\partial}{\partial t}\right) \nabla^{2} \boldsymbol{q}+\boldsymbol{g} \frac{\delta \underline{\varrho}}{\varrho_{0}} \\
&+2(\boldsymbol{q} \times \boldsymbol{\Omega}), \\
& \nabla \cdot \boldsymbol{q}=0, \\
& \frac{\partial \theta}{\partial t}+(\boldsymbol{q} \cdot \nabla) T=\kappa \nabla^{2} \theta .
\end{aligned}
$$

Within the framework of Boussinesq approximation, (6)-(8) becomes

$$
\begin{aligned}
& \frac{\partial}{\partial t} \nabla^{2} w-g \alpha\left(\frac{\partial^{2} \theta}{\partial x^{2}}+\right.\left.\frac{\partial^{2} \theta}{\partial y^{2}}\right)+2 \Omega \frac{\partial \xi}{\partial z} \\
&=\left(v+v^{\prime} \frac{\partial}{\partial t}\right) \nabla^{4} w, \\
& \frac{\partial \xi}{\partial t}-2 \Omega \frac{\partial w}{\partial z}=\left(v+v^{\prime} \frac{\partial}{\partial t}\right) \nabla^{2} \xi \\
&\left(\frac{\partial}{\partial t}-\kappa \nabla^{2}\right) \theta=\beta w .
\end{aligned}
$$

where

$\nabla^{2}=\frac{\partial^{2}}{\partial x^{2}}+\frac{\partial^{2}}{\partial y^{2}}+\frac{\partial^{2}}{\partial z^{2}}, \quad$ and $\quad \xi=\frac{\partial v}{\partial x}-\frac{\partial u}{\partial y}$

denotes the $z$-component of vorticity.

We now analyze the disturbances into normal modes, assuming that the perturbation quantities are of the form

$[w, \theta, \xi]=[W(z), \Theta(z), Z(z)] \exp \left(i k_{x} x+i k_{y} y+n t\right)$,

where $k_{x}, k_{y}$ are wave numbers along the $x$ and $y$ directions, respectively, $k^{2}=k_{x}^{2}+k_{y}^{2}$ and $n$ is, in general, a complex constant.
Letting

$$
\begin{aligned}
& a=k d, \quad \sigma=\frac{n d^{2}}{v}, \quad p_{1}=\frac{v}{\kappa}, \\
& (x, y, z) \rightarrow(\hat{x} d, \hat{y} d, \hat{z} d) \quad \text { and } \quad F=\frac{v^{\prime}}{d^{2}},
\end{aligned}
$$

(9) -(11), using (12), give

$$
\begin{gathered}
{\left[\sigma\left(D^{2}-a^{2}\right) W+\left(\frac{g \alpha d^{2}}{v}\right) a^{2} \Theta+\left(T_{A}^{1 / 2} d\right) D Z\right]} \\
=(1+F \sigma)\left(D^{2}-a^{2}\right)^{2} W, \\
{\left[(1+F \sigma)\left(D^{2}-a^{2}\right)-\sigma\right] Z=-\frac{T_{A}^{1 / 2}}{d} D W} \\
{\left[D^{2}-a^{2}-p_{1} \sigma\right] \Theta=-\left(\frac{\beta d^{2}}{\kappa}\right) W}
\end{gathered}
$$

where $T_{A}=\frac{4 \Omega^{2} d^{4}}{v^{2}}$ denotes the Taylor number and $D=\frac{\mathrm{d}}{\mathrm{d} \hat{z}}$. Applying the operator

$$
\left[D^{2}-a^{2}-p_{1} \sigma\right]\left[(1+F \sigma)\left(D^{2}-a^{2}\right)-\sigma\right]
$$

to (13), we eliminate $\Theta$ and $Z$ to obtain

$$
\begin{aligned}
& \sigma\left(D^{2}-a^{2}\right)\left(D^{2}-a^{2}-p_{1} \sigma\right)\left[(1+F \sigma)\left(D^{2}-a^{2}\right)-\sigma\right] W \\
&- R a^{2}\left[(1+F \sigma)\left(D^{2}-a^{2}\right)-\sigma\right] W \\
&-T_{A}\left(D^{2}-a^{2}-p_{1} \sigma\right) D^{2} W \\
&=(1+F \sigma)\left(D^{2}-a^{2}-p_{1} \sigma\right)\left(D^{2}-a^{2}\right)^{2} \\
& \cdot\left[(1+F \sigma)\left(D^{2}-a^{2}\right)-\sigma\right] W,
\end{aligned}
$$

where $R=\frac{g \alpha \beta d^{4}}{v \kappa}$ stands for Rayleigh number.

We now assume that the fluid layer is confined between two free boundaries. The case is of artificial nature, but due to mathematical simplicity it enables us to show the behaviour of rotation on the RivlinEricksen elastico-viscous fluid analytically. Then the boundary conditions appropriate for the problem are

$$
\begin{aligned}
& W=D^{2} W=0, \quad \Theta=0, \\
& D Z=0 \quad \text { at } \quad \hat{z}=0 \quad \text { and } \hat{z}=1 .
\end{aligned}
$$

Dropping the caps for convenience and using the above boundary conditions, it can be shown that all the even order derivatives of $W$ must vanish on the boundaries, and hence the proper solution of (16) characterizing the lowest mode is

$$
W=W_{0} \sin \pi z,
$$

where $W_{0}$ is a constant. 
Substituting (18) in (16), we obtain the dispersion relation

$(1+x)\left(1+x+i p_{1} \sigma_{1}\right)\left\{\left(1+i \sigma_{1} \pi^{2} F\right)(1+x)+i \sigma_{1}\right\}$

$R_{1}=\frac{\left[\left(1+i \sigma_{1} \pi^{2} F\right)(1+x)+i \sigma_{1}\right]+T_{1}\left(1+x+i p_{1} \sigma_{1}\right)}{x\left[\left(1+i \sigma_{1} \pi^{2} F\right)(1+x)+i \sigma_{1}\right]}$,

where

$R_{1}=\frac{R}{\pi^{4}}, \quad T_{1}=\frac{T_{A}}{\pi^{4}}, \quad a^{2}=\pi^{2} x, \quad \frac{\sigma}{\pi^{2}}=i \sigma_{1}$,

it being remembered that $\sigma$ can be complex.

Here we consider the overstable mode and so $\sigma_{1}$ is real in (19).

For the case of stationary convection, i.e. $\sigma=0,(19)$ reduces to

$$
R_{1}=\frac{(1+x)^{3}+T_{1}}{x} .
$$

We thus find that for the stationary convection, the visco-elasticity parameter $F$ vanishes with $\sigma$, and the Rivlin-Ericksen elastico-viscous fluid behaves like an ordinary Newtonian fluid.

To study the effect of rotation, we examine the nature of $\mathrm{d} R_{1} / \mathrm{d} T_{1}$.

From (19), it follows that

$$
\frac{\mathrm{d} R_{1}}{\mathrm{~d} T_{1}}=\frac{\left(1+x+i p_{1} \sigma_{1}\right)}{x\left[\left(1+i \sigma_{1} \pi^{2} F\right)(1+x)+i \sigma_{1}\right]} .
$$

On equating the real and imaginary parts of (21), it follows that

$$
\frac{\mathrm{d} R_{1}}{\mathrm{~d} T_{1}}=\frac{1}{x},
$$

which is always positive. The rotation thus has a stabilizing influence.

\section{Stability of the System and Oscillatory Modes}

Multiplying (13) by $W^{*}$, the complex conjugate of $W$, integrating over the range of $z$ and making use of (14) and (15) together with the boundary conditions (17), we obtain

$$
\begin{aligned}
-\sigma I_{1}+\frac{g \alpha \kappa a^{2}}{v \beta} & \left(I_{2}+p_{1} \sigma^{*} I_{3}\right)-d^{2}\left(1+F \sigma^{*}\right) I_{4} \\
- & d^{2} \sigma^{*} I_{5}=(1+F \sigma) I_{6},
\end{aligned}
$$

where

$$
\begin{aligned}
& I_{1}=\int_{0}^{1}\left(|D W|^{2}+a^{2}|W|^{2}\right) \mathrm{d} z \\
& I_{2}=\int_{0}^{1}\left(|D \Theta|^{2}+a^{2}|\Theta|^{2}\right) \mathrm{d} z \\
& I_{3}=\int_{0}^{1}|\Theta|^{2} \mathrm{~d} z \\
& I_{4}=\int_{0}^{1}\left(|D Z|^{2}+a^{2}|Z|^{2}\right) \mathrm{d} z \\
& I_{5}=\int_{0}^{1}|Z|^{2} \mathrm{~d} z \\
& I_{6}=\int_{0}^{1}\left(\left|D^{2} W\right|^{2}+2 a^{2}|D W|^{2}+a^{4}|W|^{2}\right) \mathrm{d} z,
\end{aligned}
$$

and $\sigma^{*}$ is the complex conjugate of $\sigma$. The integrals $I_{1}-I_{6}$ are all positive definite. Putting $\sigma=\sigma_{\mathrm{r}}+i \sigma_{\mathrm{i}}$ in (23) and equating the real and imaginary parts, we obtain

$$
\begin{array}{r}
\sigma_{\mathrm{r}}\left[-I_{1}+\frac{g \alpha \kappa a^{2}}{v \beta}\right. \\
\left.p_{1} I_{3}-d^{2} F I_{4}-d^{2} I_{5}-F I_{6}\right] \\
=-\frac{g \alpha \kappa a^{2}}{v \beta} I_{2}+d^{2} I_{4}+I_{6}
\end{array}
$$

and

$$
\sigma_{\mathrm{i}}\left[I_{1}+\frac{g \alpha \kappa a^{2}}{v \beta} p_{1} I_{3}-d^{2} F I_{4}-d^{2} I_{5}+F I_{6}\right]=0 \text {. }
$$

It is evident from (25) that $\sigma_{\mathrm{r}}$ is positive or negative. The system is therefore stable or unstable. It is clear from (26) that rotation and viscoelasticity introduce oscillatory modes in the system which were non-existent in their absence.

\section{The Case of Overstability}

Here we discuss whether instability may occur as an overstability. When the marginal state is oscillatory, we must have $\sigma_{\mathrm{r}}=0, \sigma_{\mathrm{i}} \neq 0$.

Since for overstability we wish to determine the critical Rayleigh number for the onset of instability via a state of pure oscillations, it will suffice to find conditions for which (19) will allow for solutions with $\sigma_{1}$ real. Separating the real and imaginary parts of (19), we have

$$
\begin{aligned}
R_{1}(\alpha-1)= & \alpha^{3}-\alpha \sigma_{1}^{2}\left[\left(1+\pi^{2} F \alpha\right)\right. \\
& \left.\cdot\left(1+\pi^{2} F \alpha+2 p_{1}\right)\right]+T_{1},
\end{aligned}
$$




$$
\begin{aligned}
& R_{1}(\alpha-1)\left(1+\pi^{2} F \alpha\right)=\left(1+\pi^{2} F \alpha\right) \\
& \cdot \alpha\left[2 \alpha^{2}-\sigma_{1}^{2} p_{1}\left(1+\pi^{2} F \alpha\right)\right]+p_{1}\left(\alpha^{3}+T_{1}\right),
\end{aligned}
$$

where $1+x=\alpha$.

Eliminating $R_{1}$ between (27) and (28), we have

$$
\begin{aligned}
& {\left[\pi^{4} F \alpha^{2}\left(p_{1}+\pi^{2} F^{2} \alpha\right)+\left(1+\pi^{2} F \alpha\right)\left(p_{1}+3 \pi^{2} F \alpha\right)\right.} \\
& \left.\quad+\left(1+p_{1} \pi^{2} F \alpha\right)\right] \alpha \sigma_{1}^{2}+\left[\pi^{2} F \alpha^{4}+\left(1+p_{1}\right) \alpha^{3}\right. \\
& \left.\quad+T_{1}\left(p_{1}-1-\pi^{2} F \alpha\right)\right]=0 .
\end{aligned}
$$

Equation (29) is of the form

$$
A \sigma_{1}^{2}+B=0,
$$

where $A, B$ are coefficients of $\sigma_{1}^{2}$ and a constant term, respectively, in (29), and $A$ is positive.

[1] S. Chandrasekhar, Hydrodynamic and Hydromagnetic Stability, Clarendon Press, Oxford 1961, Chapt. III.

[2] P. K. Bhatia and J. M. Steiner, Z. Angew. Math. Mech. 52, 321 (1972).

[3] R. C. Sharma, Acta Phys. Hung. 40, 11 (1976).

[4] J. G. Oldroyd, Proc. Roy. Soc. (London) A245, 278 (1958).
Since $\sigma_{1}$ is real for overstability, the value of $\sigma_{1}^{2}$ is positive. Equation (30) shows that this is impossible if $B>0$. Therefore $B>0$ gives a sufficient condition for the non-existence of overstability, which yields

$$
p_{1}>1+\pi^{2} F \alpha,
$$

which further implies that

$$
\frac{v}{\kappa}>1+F\left(\pi^{2}+k^{2} d^{2}\right) .
$$

The condition $\frac{v}{\kappa}>1+F\left(\pi^{2}+k^{2} d^{2}\right)$ is, therefore, a sufficient condition for the non-existence of overstability, the violation of which does not necessarily imply occurrence of overstability.

[5] R. K. Srivastava and K. K. Singh, Bull. Cal. Math. Soc. 80, 286 (1988).

[6] A. Garg, R. K. Srivastava, and K. K. Singh, Proc. Nat. Acad. Sci. India 64 A, 355 (1994). 